\title{
ON A UNIVERSAL MECHANISM FOR LONG RANGED VOLATILITY CORRELATIONS
}

\author{
Jean-Philippe Bouchaud ${ }^{1,2}$, Irene Giardina ${ }^{3}$ and Marc Mézard ${ }^{4}$ \\ 1 Service de Physique de l'État Condensé, Centre d'études de \\ Saclay, \\ Orme des Merisiers, 91191 Gif-sur-Yvette Cedex, France \\ 2 Science \& Finance, 109-111 rue Victor-Hugo, 92532 France \\ 3 Service de Physique Théorique, Centre d'études de Saclay, \\ Orme des Merisiers, 91191 Gif-sur-Yvette Cedex, France \\ ${ }^{4}$ Laboratoire de Physique Théorique et Modèles Statistiques \\ Université Paris Sud, Bat. 100, 91405 Orsay Cedex, France
}

\begin{abstract}
We propose a general interpretation for long-range correlation effects in the activity and volatility of financial markets. This interpretation is based on the fact that the choice between 'active' and 'inactive' strategies is subordinated to random-walk like processes. We numerically demonstrate our scenario in the framework of simplified market models, such as the Minority Game model with an inactive strategy. We show that real market data can be surprisingly well accounted for by these simple models.
\end{abstract}

A well documented 'stylized fact' of financial markets is volatility clustering [1, 2, 3, [4]. Figure 1 compares the time series of the daily returns of the Dow-Jones index since 1900 and that of a Brownian random walk. Two features are immediately obvious to the eye: the volatility does indeed 
have rather strong intermittent fluctuations, and these fluctuations tend to persist in time. A more quantitative analysis shows that the daily volatility $\sigma_{t}$ (defined, for example, as the average squared high frequency returns) has a log-normal distribution [6], and that its temporal correlation function $\left\langle\sigma_{t} \sigma_{t+\tau}\right\rangle$ can be fitted by an inverse power of the lag $\tau$, with a rather small exponent in the range $0.1-0.3$ [2, 5, 6, 7]. This suggests that there is no characteristic time scale for volatility fluctuations: outbursts of market activity can persist for rather short times (say a few days), but also for much longer times, months or even years. A very interesting observation is that these long ranged volatility correlations are observed on many different financial markets, with qualitatively similar features: stocks, currencies, commodities or interest rates. This suggests that a common mechanism is at the origin of this rather universal phenomenon.

A first possibility is that the apparent lack of time scale associated with the power-law dependence of the correlation function is a consequence of the fact that human activity is naturally rythmed by days, weeks, months, quarters and years. Now, the ratio between these successive time scales is roughly constant. The superposition of correlation functions with time constants uniformly distributed on a log-scale may easily be confused with a single power-law with a small exponent 8].

However, very important insights into market dynamics have recently been gained by the study of several agent based models [9, 10, 11, 12, 13, 14, 10, 15, 16. These models postulate some simple behaviour at the level of the agents and investigate the resulting price dynamics. Among others, the model by Lux and Marchesi [14] assumes that each agent can behave, as a function of time, either as a fundamentalist (i.e. determining his action by comparing the current market price to some fundamental 'true' price), or as a 'trend follower', influenced by observed past trends on the price itself. Agents switch between the two strategies as a function of their relative performance. Numerical simulations based on this model produce quite realistic price charts. In particular, long-ranged, power-law type volatility correlations are reported. Another family of models, the 'Minority Game' (MG) and its variants [10, 17, 18, 20, 21], has recently become the focus of intense theoretical scrutiny. The Minority Game describes the behaviour of competing agents that can choose between different individual strategies as a function of their past performance. In its original version, this model is rather remote from financial markets; in particular, there is no price dynamics. Several at- 

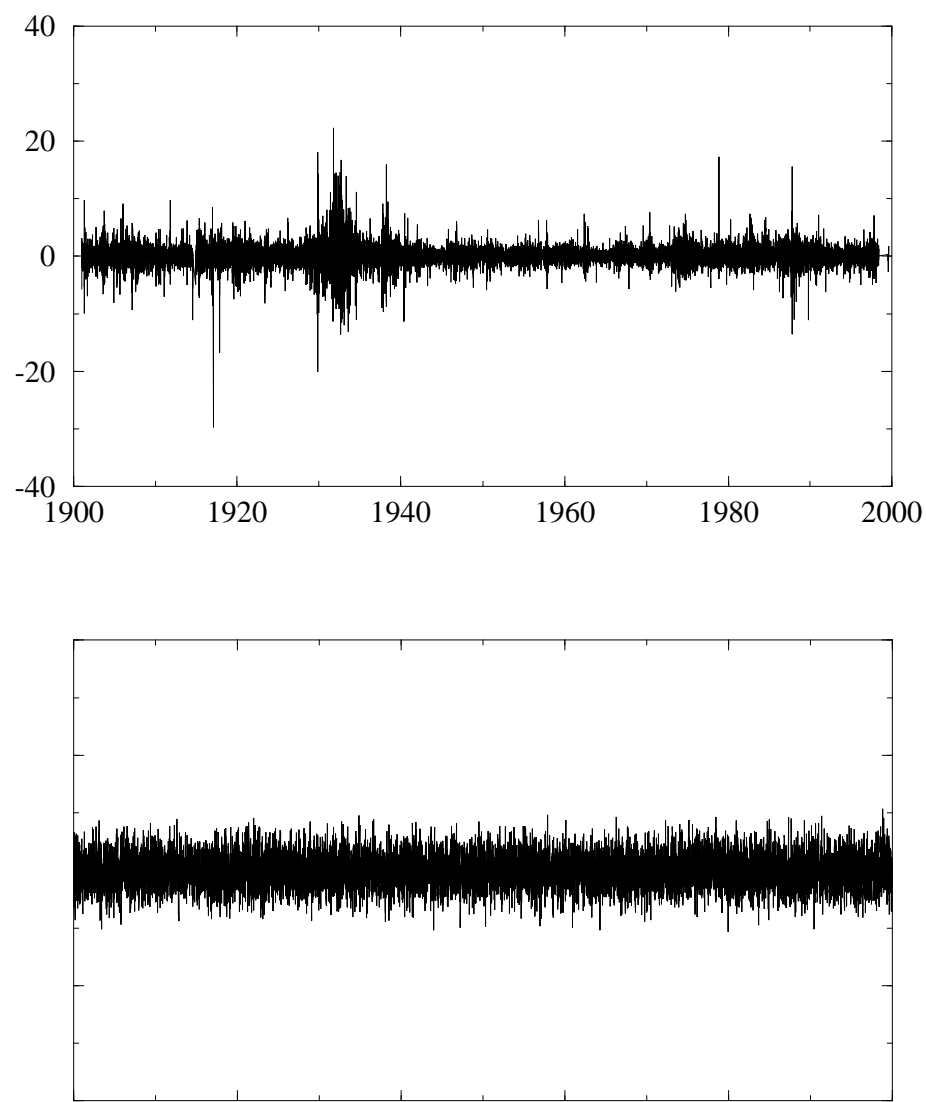

Figure 1: Top panel: daily returns of the Dow-Jones index since 1900. One very clearly sees the intermittent nature of volatility fluctuations. Note in particular the sharp feature in the 30's. Lower panel: daily returns for a Brownian random walk, showing a featureless pattern. 
tempts have been made to generalize it and construct more realistic market models [17]. As first noticed in [18, if one allows the agents to be inactive, intermittent volatility fluctuations can be generated. We have ourselves studied a market model that allows traders to switch between a bond market and a stock market, and accounts properly for their wealth balance and for market clearing. The phenomenology of this model is very rich and a detailed account of our results will be published separately 22. One of our main result is the existence of a 'turbulent' market phase where volatility fluctuations are intermittent and show a power-law correlation (see Figure 3 below). A very important point is that all these models are different in their details but all show qualitatively similar behaviour, without the explicit introduction of any of the 'human' time scales mentioned above. In other words, these agent based models assume a unique elementary time scale (say the 'day') and the long-ranged volatility correlations spontaneously emerge from the dynamics.

We wish to propose a simple and robust mechanism to account for the appearance of these long-ranged correlations in the above simplified models. We then argue that this mechanism also very naturally operates in real financial markets, and accounts well for the empirical findings. The following discussion is intentionally rather qualitative; more detailed and technical results will be presented elsewhere [22].

The idea is the following: in the above models, scores are attributed by agents to their possible strategies, as a function of their past performance. In a region of the parameter space where these models lead to an efficient market, the autocorrelation of the price increments is close to zero, which means that to a first approximation, no strategy can on average be profitable. This implies that for any reasonable definition of the update of the scores, these scores will locally behave, as a function of time, as random walks. Furthermore, the scores associated to different strategies generically behave as independent random walks. Now, in all these models, the switch between two strategies occurs when their scores cross. Therefore, in the case where each agent has two strategies, say one 'active' (trading in the market) and one 'inactive' (holding bonds), the survival time of any one of these strategies will be given by the return time of a random walk (the difference between the scores of the two strategies) to zero. The interesting point is that these return times are well known to be power-law distributed (see below): this leads to the non trivial behaviour of the volume autocorrelation function. 
In other words, the very fact that agents compare the performance of two strategies on a random signal leads to a multi-time scale situation.

More formally, let us define the quantity $\theta_{i}(t)$ that is equal to 1 if agent $i$ is active at time $t$, and 0 if inactive. The total activity is given by $a(t)=$ $\sum_{i} \theta_{i}(t)$. The time autocorrelation of the activity is given by

$$
C_{a}\left(t, t^{\prime}\right)=\left\langle a(t) a\left(t^{\prime}\right)\right\rangle=\sum_{i, j}\left\langle\theta_{i}(t) \theta_{j}\left(t^{\prime}\right)\right\rangle
$$

We will actually use in the following the so-called activity variogram, defined as:

$$
V_{a}\left(t, t^{\prime}\right)=\left\langle\left[a(t)-a\left(t^{\prime}\right)\right]^{2}\right\rangle=C_{a}(t, t)+C_{a}\left(t^{\prime}, t^{\prime}\right)-2 C_{a}\left(t, t^{\prime}\right) .
$$

One can consider two extreme cases which lead to the same result, up to a multiplicative constant: (a) agents follow completely different strategies and have independent activity patterns, i.e. $\left\langle\theta_{i} \theta_{j}\right\rangle \propto \delta_{i, j}$ or (b) agents follow very similar strategies, for example by all comparing the perfomance of stocks to that of bonds, in which case $\theta_{i}=\theta_{j}$. In both cases, one has $C_{a}\left(t, t^{\prime}\right)$ is proportional to $\left\langle\theta_{i}(t) \theta_{i}\left(t^{\prime}\right)\right\rangle$. This quantity can be computed in terms of the distribution $P(s)$ of the survival time $s$ of the strategies (in the following, we assume that both the inactive and active strategies have the same survival time distribution). Two cases must be distinguished: if $P(s)$ has a finite first moment $\langle s\rangle$ (finite average lifetime of the strategies), then $C_{a}\left(t, t^{\prime}\right)$ is stationary, i.e. it only depends on the difference $\tau=t^{\prime}-t$. Introducing the Laplace transforms $\mathcal{L} C_{a}(E)$ and $\mathcal{L} P(E)$ of $C_{a}(\tau)$ and $P(s)$, the general relation between the two quantities reads [23]:

$$
E \mathcal{L} C_{a}(E)=\left(1-\frac{2[1-\mathcal{L} P(E)]}{\langle s\rangle E[1+\mathcal{L} P(E)]}\right) .
$$

If one the other hand $P(s)$ has an infinite first moment, then $C_{a}\left(t, t^{\prime}\right)$ depends both on $t$ and $t^{\prime}$ : this is known as the aging phenomenon [24, 23]. For an unconfined random walk, the return time distribution decays as $s^{-3 / 2}$ for large $s$ and therefore its first moment is infinite. However, in all the models mentioned above, there exist 'restoring' forces which effectively confine the scores to a finite interval [22]. This can be attributed, both in the case of the MG or of more realistic market models, to 'market impact', which means

\footnotetext{
${ }^{1} \mathrm{Up}$ to an additive constant which disappears from the variogram.
} 
that good strategies tend to deteriorate because of their very use. There are many reasons to believe that such confining forces also operate in financial markets. The consequence of these effects is to truncate the $s^{-3 / 2}$ tail for values of $s$ larger than a certain equilibrium time $s_{0}$. Therefore, the first moment of $P(s)$ actually exists, such that Eq. (3) is valid. Nevertheless, one can see from Eq. (3) that the characteristic $s^{-3 / 2}$ behaviour of $P(s)$ for short time scales leads to $E \mathcal{L} C_{a}(E) \sim 1-B / \sqrt{E}+\ldots$ for $s_{0}^{-1} \ll E \ll 1$. This in turn leads to a singular behaviour for the variogram $V_{a}(\tau)$ at small $\tau$ 's, as $V_{a}(\tau) \propto \sqrt{\tau}$, before saturating to a finite value for $\tau \sim s_{0}$. Intuitively, this means that the probability for the activity to have changed significantly between $t$ and $t+\tau$ is proportional to $\int_{0}^{\tau} d s s P(s) \propto \sqrt{\tau}$ (for $\tau \ll s_{0}$ ), where $s P(s)$ is the probability to be at time $t$ playing a strategy with lifetime $s$.

Let us illustrate this general scenario with the example of the MG with an inactive strategy, first introduced in [18]. Each agent has a certain number of fixed strategies to choose from. A strategy is a mapping from a signal (for example the past history) into a decision, say +1 or -1 . The aim of the game at each time step is to make the decision that is chosen by the minority of the agents at that time [10]. If a strategy is successful (or would have been if it had been played), its score increases, conversely, if the wrong decision is made (or again, would have been if it had been played), the score decreases. The chosen strategy is the one that has the highest score. If all the strategies of an agent have negative scores, then the agent does not play. The relevant parameter $\alpha$ of this model is the ratio of the number of possible histories to the number $N$ of agents. The history is given by the $M$ past steps of the game, therefore $\alpha=2^{M} / N$. One finds [19, 22] that there is a critical value $\alpha_{c}$ above which all agents finally become inactive. Below this value, the activity is non zero. A plot of the activity as a function of time in this model is given in Figure 2, for a value of $\alpha$ smaller than $\alpha_{c}$. In the inset, we have plotted the activity variogram $V_{a}(\tau)$, which reveal the characteristic $\sqrt{\tau}$ singularity discussed above, before saturating for large $\tau\left(\sim s_{0}\right)$. This $\sqrt{\tau}$ singularity is present in the whole active phase $\alpha<\alpha_{c}$, although $s_{0}$ is large compared to 1 only if $\alpha$ is not too small. Very similar variograms have also been found in the more realistic market model that we have investigated, showing the universality of this result (see Figure 3) [22]. Note that a similar mechanism might also be present in the Lux-Marchesi model, where it has been observed that the activity bursts are associated to a large number of agents switching from being 'fundamentalists' to being 'trend followers' [14. 


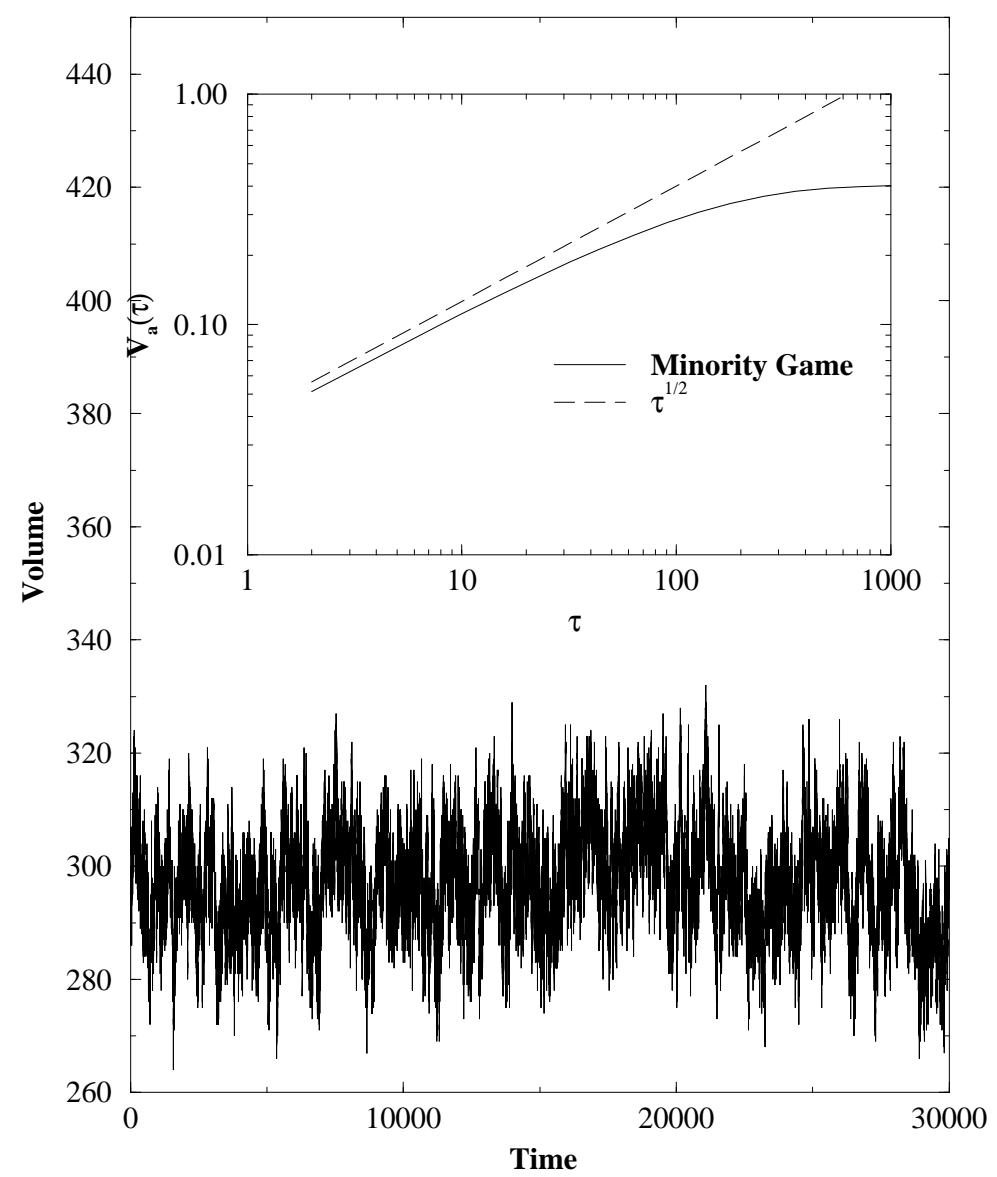

Figure 2: Volume of activity (number of active agents) as a function of time in the MG with two active strategies and one inactive strategy per agent, and for $\alpha=0.51\left(\alpha_{c} \simeq 1\right.$.). The number of agents is 501. Inset: The corresponding activity variogram as a function of the lag $\tau$, in a $\log$-log plot to emphasize the $\sqrt{\tau}$ singularity at small $\tau$ 's. 


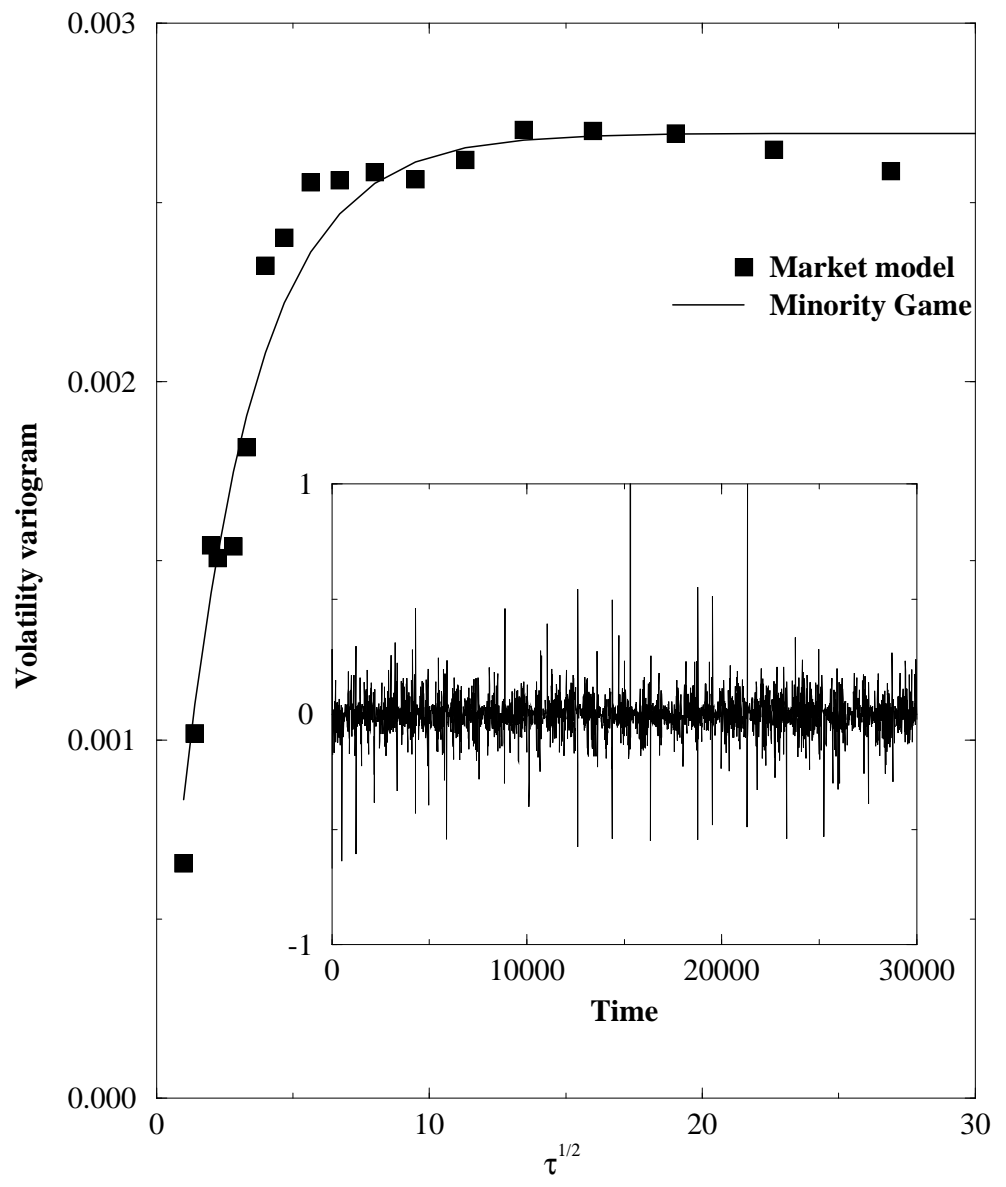

Figure 3: Volatility variogram (squares) for a market model, inspired from the MG, where agents buy and sell a stock, and can switch from the stock market to bonds. The full line corresponds to the prediction of the simple MG with an inactive strategy. Inset: Price changes as a function of time in the same model, showing volatility clustering. 
It is interesting to compare the above results with real market data. Figure 4 shows the volume of activity on the S\&P 500 futures contract in the years 1985-1998. This plot is to the eye very similar to the one of Figure 3, obtained with the MG. This is quantitatively confirmed by the activity variogram, shown in the inset. On the same graph, we have reproduced the MG result. Both the time scale and the volume scale (arbitrary in the MG model) have been adjusted to get the best agreement. Furthermore, a constant has been added to $V_{a}$ (corresponding to a $\delta_{\tau, 0}$ contribution to $C_{a}$ ), to account for the fact that part of the trading activity is certainly white noise (e.g. motivated by news, or by other non strategic causes). As can be seen, the agreement is rather good. Most significant is the clear $\sqrt{\tau}$ behaviour at small $\tau(\tau<50$ days). We therefore suggest that the effect captured by the MG (Figure 2) or more sophisticated variants (Figure 3), namely the subordination of the activity on random walk like signals, is also present in real markets. It seems to us that this makes perfect sense since market participants indeed compare the results of different strategies to decide whether they should remain active in a market or leave it.

Since the volatility and the volume of activity are strongly correlated in financial markets [25, 26], our interpretation should naturally carries over to volatility fluctuations as well. This is illustrated in Figure 5, where the variogram of the log-volatility for major stock indices is shown, together with the very same MG result. Again, the agreement is very good. We have also shown for comparison the prediction of the multifractal model of ref. [0], $V_{a}(\tau)=2 \lambda^{2} \log \left(\tau / \tau_{0}\right)$. It is interesting to note that the two model, although very different, lead to nearly indistinguishable numerical fits.

The analogy between volatility clustering in financial markets and intermittency effects in turbulent flows has recently been emphasized [27, 0, 28]. It is tempting to speculate that the mechanism discussed here might also be at work in turbulent flows, where outburts of activity are due to localized structures [29]. If the motion of these localized structures locally resembles that of a random walk, similar conclusions can be expected.

In summary, we have proposed a very general interpretation for longrange correlation effects in the activity and volatility of financial markets. This interpretation is based on the fact that the choice between different strategies is subordinated to random-walk like processes. We have numerically demonstrated our scenario in the framework of simplified market models, and showed that, somewhat surprisingly, real market data can actually 


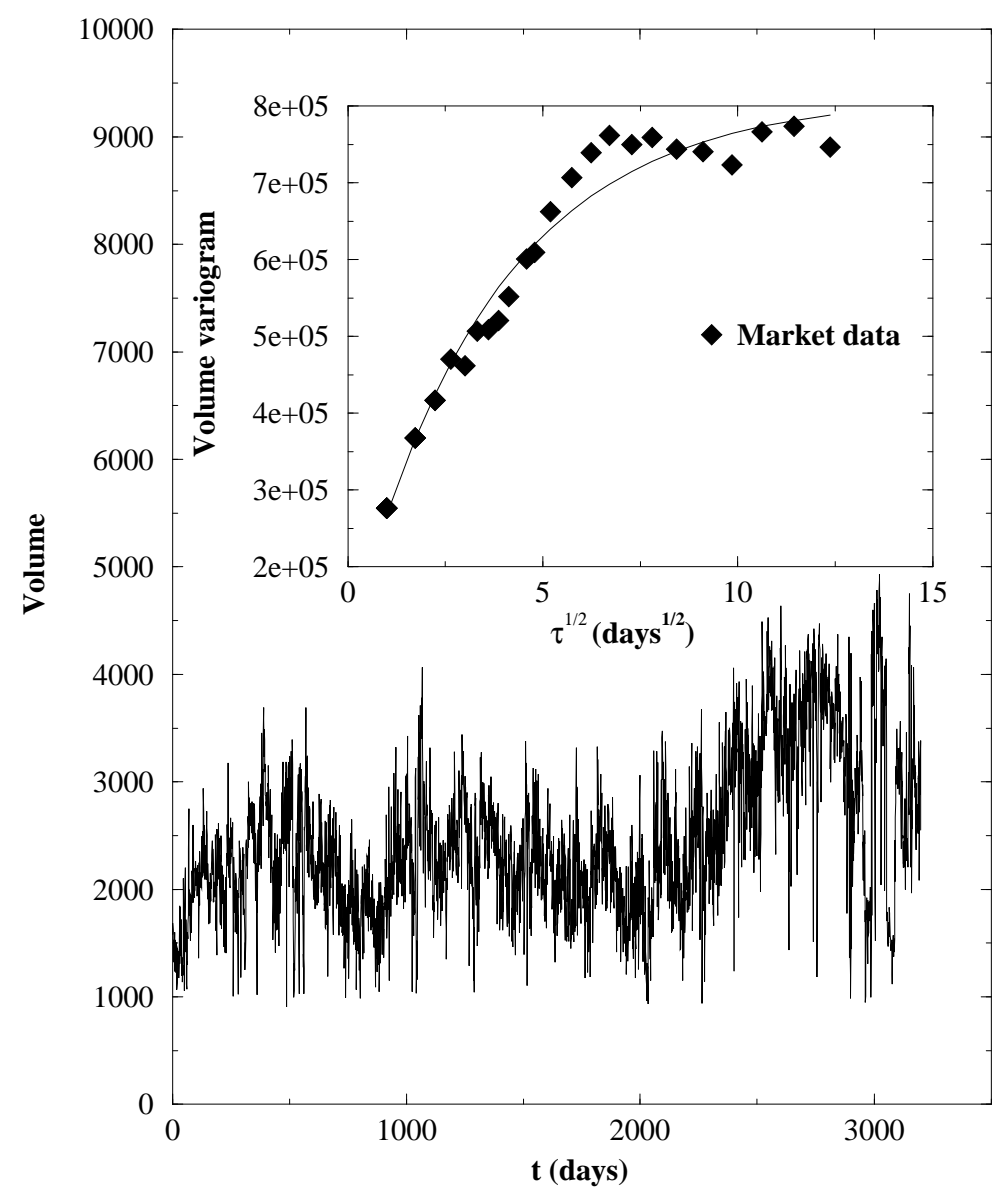

Figure 4: Daily volume of activity (number of trades) on the S\&P 500 in the years 1985-1998: compare to Figure 2. Inset: Corresponding variogram (diamonds) as a function of the square-root of the lag. Note the clear linear behaviour for small $\sqrt{\tau}$. The full line is the MG result, with both axis rescaled and a constant added to account for the presence of 'white noise' trading. 


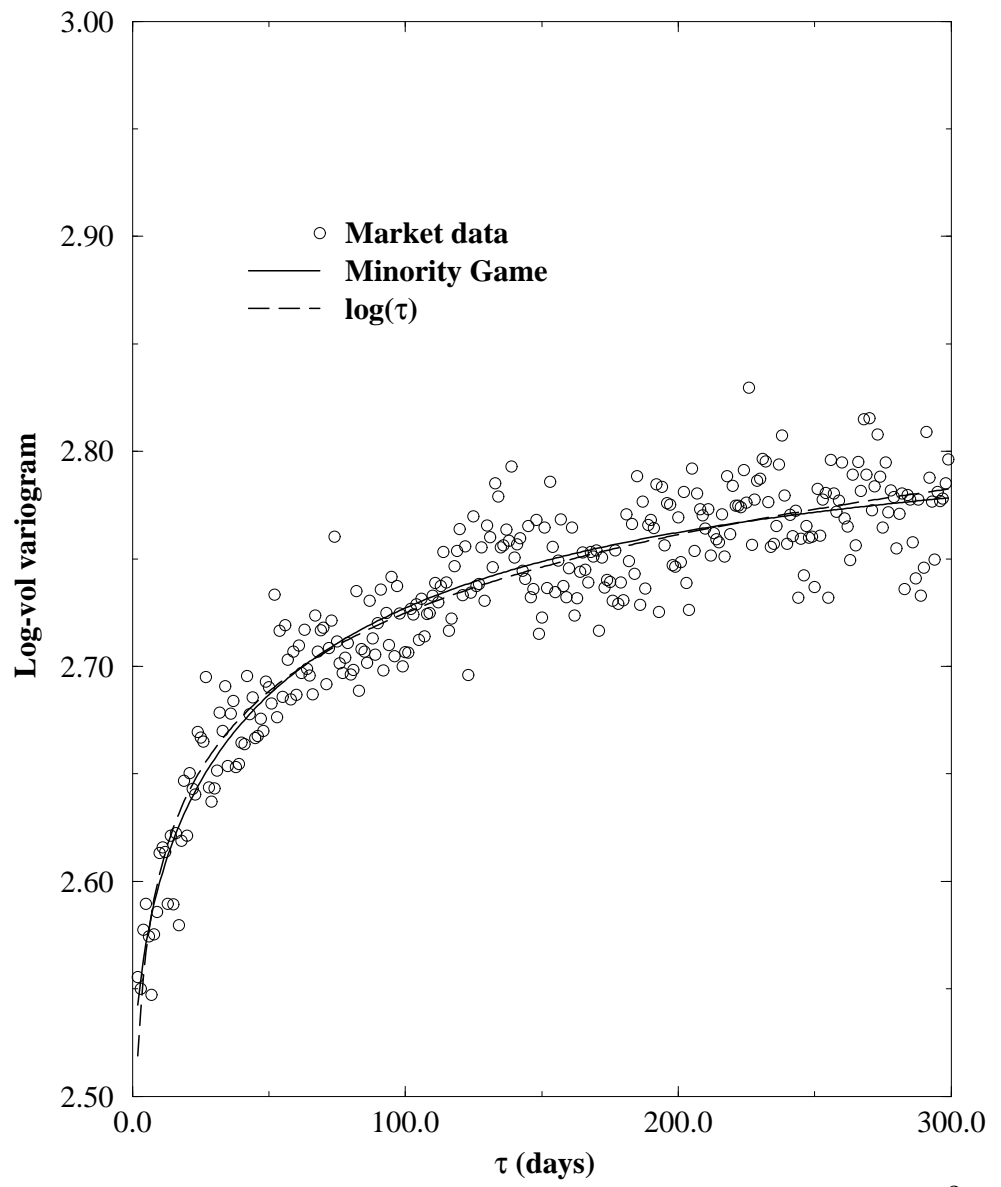

Figure 5: Variogram of the log-volatility, $\left\langle\log ^{2}\left(\sigma_{t} / \sigma_{t+\tau}\right)\right\rangle$ as a function of $\tau$, averaged over 17 different stock indices (American, European, Asian). The full line is the MG result, with again both axis rescaled and a constant added to account for the presence of 'white noise' trading. The dashed line is the prediction of the multifractal model of [7], and is nearly indistinguishable from the MG result. 
be quite accurately accounted for by these simple models (see Figs 4 and 5).

Acknowledgements We wish to thank M. Potters for several important discussion on this topic, and A. Matacz and Ph. Seager for providing the data of Figs 4 and 5, and for interesting remarks. Useful interactions with G. Canat, D. Farmer, E. Moro, J.P. Garrahan, N. Johnson, M. Marsili, D. Sherrington and H. Zytnicki are acknowledged.

\section{References}

[1] A. Lo, Econometrica, 59, 1279 (1991).

[2] Z. Ding, C. W. J. Granger and R. F. Engle, J. Empirical Finance 1, 83 (1993).

[3] R. Mantegna \& H. E. Stanley, An introduction to Econophysics, Cambridge University Press, 1999.

[4] J.-P. Bouchaud and M. Potters, Théorie des Risques Financiers, AléaSaclay, 1997; Theory of Financial Risks, Cambridge University Press, 2000 .

[5] R. Cont, M. Potters, J.-P. Bouchaud, Scaling in stock market data: stable laws and beyond, in Scale invariance and beyond, B. Dubrulle, F. Graner, D. Sornette (Edts.), EDP Sciences (1997); M. Potters, R. Cont, J.-P. Bouchaud, Europhys. Lett. 41, 239 (1998).

[6] Y. Liu, P. Cizeau, M. Meyer, C.-K. Peng, H. E. Stanley, Physica A245 437 (1997); P. Cizeau, Y. Liu, M. Meyer, C.-K. Peng, H. E. Stanley, Physica A245 441 (1997).

[7] A. Arnéodo, J.-F. Muzy, D. Sornette, Eur. Phys. J. B 2, 277 (1998); J.-F. Muzy, J. Delour, E. Bacry, e-print cond-mat/0005400.

[8] J.-P. Bouchaud, Power in Economy and Finance, some ideas from physics, Quantitative Finance, 1, xxx, (2000)

[9] P. Bak, M. Paczuski, and M. Shubik, Physica A 246, 430 (1997) 
[10] D. Challet, Y.-C. Zhang, Physica A246, 407 (1997)

[11] J.-P. Bouchaud, R. Cont, European Journal of Physics B 6, 543 (1998); R. Cont, J.P. Bouchaud, Macroeconomics Dynamics, 4, 170 (2000).

[12] J.D. Farmer, Market Force, Ecology and Evolution, e-print adaporg/9812005, Int. J. Theo. Appl. Fin. 3, 425 (2000).

[13] D. Sornette, D. Stauffer, H. Takayasu, Market fluctuations, multiplicative and percolation models, size effects and predictions, e-print condmat/9909439, and references therein.

[14] T. Lux, M. Marchesi, Nature 397, 498 (1999), Int. J. Theo. Appl. Fin. 3, 675 (2000).

[15] G. Iori, A microsimulation of traders activity in the stock market: the role of heterogeneity, agents' interactions and trade frictions, e-print adap-org/9905005.

[16] For a review, see J. D. Farmer, Physicists attempt to scale the ivory towers of finance, in Computing in Science and Engineering, November 1999, reprinted in Int. J. Theo. Appl. Fin. 3, 311 (2000).

[17] D. Challet, M. Marsili, Y.-C. Zhang, Modeling Market Mechanism with Minority Game, e-print cond-mat/9909265. D. Challet, M. Marsili, R. Zecchina, Int. J. Theo. Appl. Fin. 3, 451 (2000).

[18] P. Jefferies, M. Hart, P.M. Hui, N. F. Johnson, From market games to real world markets, e-print cond-mat/0008387; Int. J. Theo. Appl. Fin. 3, 443 (2000).

[19] G. Canat, H. Zytnicki, M. Mézard, Ecole Polytechnique Internal Report (June 2000).

[20] A. Cavagna, J.P. Garrahan, I. Giardina, D. Sherrington, Phys. Rev. Lett. 83, 4429 (1999).

[21] E. Moro, J.P. Garrahan, D. Sherrington, Phys. Rev. E62, R9 (2000).

[22] I. Giardina, J.P. Bouchaud, M. Mézard, in preparation. 
[23] C. Godrèche, J.M. Luck, Statistics of the occupation time of renewal processes, e-print cond-mat/0010428

[24] for a review, see J.P. Bouchaud, L. Cugliandolo, J. Kurchan, M. Mézard, Out of Equilibrium dynamics in spin-glasses and other glassy systems, in 'Spin-glasses and Random Fields', A.P. Young Editor (World Scientific) 1998, and references therein.

[25] G. Bonnano, F. Lillo, R. Mantegna, Dynamics of the number of trades in financial securities, e-print cond-mat/9912006

[26] V. Plerou, P. Gopikrishnan, L.A. Amaral, X. Gabaix, H.E. Stanley, eprint cond-mat/9912051.

[27] S. Ghashghaie, W. Breymann, J. Peinke, P. Talkner, Y. Dodge, Nature 381767 (1996).

[28] J.P. Bouchaud, M. Potters, M. Meyer, Eur. Phys. J. B 13, 595 (1999).

[29] U. Frisch, Turbulence: The Legacy of A. Kolmogorov, Cambridge University Press (1997). 